\title{
STRATEGIES PAYSANNES DE CONSERVATION DE QUELQUES RESSOURCES PHYTOGENETIQUES DANS LE MOYEN-MONO AU TOGO
}

\author{
S. AKPAVI', M. BANOIN ${ }^{2}$, K. BATAWILA ${ }^{1}$, R. VODOUHE $^{3}$ et K. AKPAGANA ${ }^{1}$ \\ ${ }^{1}$ Laboratoire de Botanique et Ecologie végétale, Faculté des Sciences, Université de Lomé, B. P. 1515, Lomé (Togo). \\ E-mail : benakpavi@yahoo.fr \\ ${ }^{2}$ Faculté d'Agronomie, Université Abdou Moumouni, B. P. 10960, Niamey (Niger) \\ ${ }^{3}$ Institut International pour les Ressources Phytogénétiques (IPGRI), Bureau Régional pour l'Afrique de l'Ouest \\ et du Centre, c/o IITA, B. P. 08-0932, Cotonou
}

\begin{abstract}
RESUME
L'étude, réalisée dans le Moyen-mono (Sud-Est du Togo), analyse les pratiques paysannes de conservation des ressources phytogénétiques. Les pratiques agricoles sont évaluées selon leur modalité, leur opportunité et leur efficacité à travers des interviews semi-structurées et des observations directes dans les champs et les ménages. Les pratiques de conservation des semences les plus courantes observées sont l'utilisation des greniers de types "Huidza» et "Ava» pour le maïs, la mise en bidons et fûts pour le niébé. Des produits sont utilisés pour renforcer la conservation. II s'agit de la cendre, du jus d' Azadirachta indica, du sable fin, des grains de sel de cuisine, de la sciure de bois pour le maïs ; du péricarpe sec de Citrus $s p$. et/ou du Capsicum sp. pour le niébé. Des bottes de tiges de manioc sont mises contre tuteurs en milieu ombragé alors que les semences d'ignames sont conservées en clôture à domicile ou en fosse au champ. Ces pratiques, très efficaces, portant moins atteinte à l'environnement doivent être encouragées.
\end{abstract}

Mots clés : Togo, Moyen-Mono, ressources phytogénétiques, pratiques de conservation.

ABSTRACT

FARMERS'STRATEGIES FOR PHYTOGENETIC RESOURCES CONSERVATION IN THE MIDDLE MONO (TOGO)

The study undertaken in Middle-Mono, South-east Togo, aims to analyse farmers conservation practices of PhytoGenetic Resources. Agricultural practices were assessed according type, opportunity and effectiveness, through semi-structured household interviews and direct farm observations. The main practices of seed conservation are the use of "Huidza» and "Ava» granaries for maize, cask or bole and baril for cowpea. Some homemade products are added to seeds in order improve conservation; these are kitchen salt, woodash, sawdust, fine sand, Azadirachta indica juice, for maize and dry pericarp of Citrus sp. and/or Capsicum sp. for cowpea. Top boots of cassava are staked while yam seeds are put into pits in farm or in a barn at home. As the practices of phytogenetic resources conservation in Moyen-Mono are found to be efficient and have less environmental adverse effects, their use by farmes must be encouraged.

Keywords : Togo, Moyen-mono, phytogenetic resources, farm conservation.

\section{INTRODUCTION}

Les ressources phytogénétiques sont définies comme étant l'ensemble des génotypes végétaux utilisés ou potentiellement utilisables par l'homme. Elles conditionnent la durabilité des systèmes de production et constituent les clés du développement (Engels et al., 2001 ;
Weiskopf, 2001). En outre, les ressources phytogénétiques constituent l'élément fondamental de la survie des populations de par le monde.

On note, malheureusement, que la destruction des ressources naturelles liée à la pression sur les terres et aux pratiques agricoles inappropriées contribue à l'érosion de ces ressources phytogénétiques (Groupe Crucible II, 2001). 
Au Togo, la diversité des ressources phytogénétiques pour l'alimentation et l'agriculture est bien fournie. Les principales ressources utilisées sont les céréales, les légumineuses à graines, les plantes à racines et tubercules, les légumes, les condiments et épices, les oléagineux, les fibres, les aromatiques, les fruitiers cultivés et sauvages, les plantes fourragères, les plantes médicinales et les champignons. Pour assurer la sécurité alimentaire durable aux populations, la préservation de ces ressources et, surtout, des semences s'avère indispensable.

Généralement, ces ressources phytogénétiques sont conservées, soit dans des banques de gènes (conservation ex situ), soit dans leurs habitats naturels (conservation in situ ou à la ferme). Engels (2001) a montré qu'il existe très peu d'investigations dans ce dernier cas. Cependant, en ce qui concerne les efforts faits pour pérenniser les ressources phytogénétiques, on reconnaît beaucoup plus aujourd'hui l'importance des agriculteurs et des populations autochtones dans la conservation, l'utilisation et l'amélioration de la diversité biologique (IPGRI, 2001). En effet, les paysans détiennent les modes d'exploitation culturaux qui favorisent le maintien de la diversité génétique en équilibre avec les écosystèmes. Aussi, ces paysans étant très attachés aux variétés traditionnelles, ils sélectionnent à chaque campagne des spécimens qu'ils conservent comme semences pour la prochaine saison culturale. Dans bien des cas, les ressources phytogénétiques sont ainsi relativement conservées par les systèmes culturaux aussi longtemps qu'elles sont utiles pour les agriculteurs.

Au Togo, plusieurs travaux ont signalé l'existence de diverses formes de savoirs traditionnels qui sous-tendent la bonne gestion des ressources phytogénétiques dans différentes régions (Kakabou, 1991 ; Knoth, 1993 ; Gnamkoulamba, 2001 ; Datchidi, 2002 ; Akpavi, 2003). La culture et les connaissances locales font partie intégrante de la biodiversité agricole car c'est l'activité agricole de l'homme qui, si elle est bien pratiquée, préserve cette biodiversité. La présente étude est une analyse des stratégies paysannes de conservation de certaines ressources phytogénétiques dans les systèmes de culture pratiqués dans la Préfecture du Moyen-Mono (Sud-Est du Togo).

\section{MATERIEL ET METHODES}

\section{ZONE D'ETUDE}

La préfecture du moyen-Mono est située dans la partie sud-est du Togo entre les latitudes $7^{\circ}$ et $8^{\circ}$ Nord (Figure 1 ). Elle couvre une superficie de $1010 \mathrm{~km}^{2}$ (Anonyme, 2002) sur des sols ferrugineux tropicaux, surexploités et érodés. Elle est drainée par le fleuve Mono. Le climat est de type guinéen avec deux saisons de pluie. La végétation est une mosaïque de forêts galeries, de savanes, de jachères et de champs dont les ligneux les plus fréquents sont Vitellaria paradoxa C. F. Gaertner, Parkia biglobosa (Jacq) R. Br. et Vitex doniana Sweet (Brunel et al., 1984).

La population du Moyen-Mono était de 65800 habitants en 1999 (Anonyme, 2002) avec une croissance annuelle de 2,96\%. Elle est à $80 \%$ agricole (DSID, 1998). Les groupes ethniques majoritaires sont les Ehoé $(61 \%)$ et les Adja (38\%).

Les cultures vivrières sont les céréales [maïs (Zea mays L.) surtout], les tubercules [essentiellement manioc (Manihot esculenta Crantz) et igname (Dioscorea spp.)], le palmier à huile (Elaeis guineensis L.) et les légumineuses. Les berges des cours d'eau permettent aux paysans de faire, accessoirement, des cultures maraîchères de contre saison comme la tomate (Lycopersicum esculentum L.), le piment (Capsicum spp.), la laitue (Latuca sativa L.) et le gombo (Abelmoschus esculentus Moench).

L'agriculture est encore traditionnelle dans la zone avec diverses pratiques locales de conservation des récoltes.

La localité concernée est le canton de Tohoun, chef-lieu de la préfecture du Moyen-Mono. II compte cinq quartiers dont Tohoun Kpoenzouhoé qui a servi de base dans le cadre de cette étude. Une enquête préliminaire a montré que ce quartier comprend 95 exploitations agricoles dont 38 représentatives ont été investiguées, soit un taux de sondage de $40 \%$. 


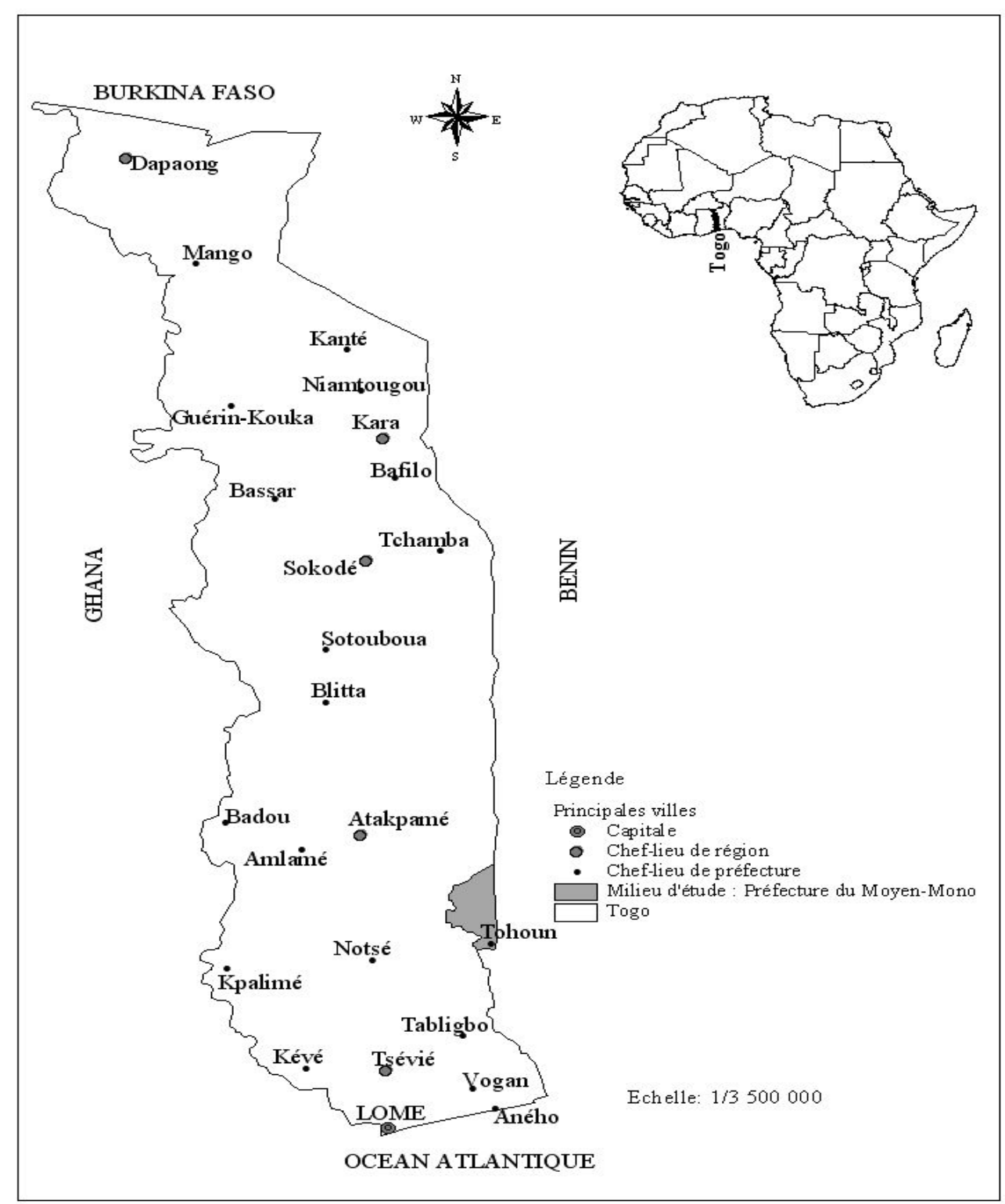

Figure 1 : Localisation du Moyen-Mono.

Situation of Middle Mono in Togo.

\section{ECHANTILLONNAGE ET COLLECTE DES DONNEES}

La situation agricole est supposée globalement quasi homogène entre les cinq quartiers de la ville de Tohoun. La diversité des pratiques agricoles du milieu pourrait alors être évaluée à l'intérieur d'un quartier.

La démarche méthodologique a consisté en un échantillonnage en grappes. L'échantillonnage est en grappes du fait du choix. Un seul quartier a été retenu : Kpoenzouhoé. Ce quartier est le plus ancien de la ville de Tohoun. C'est aussi le quartier royal et le quartier où cohabitent en parfaite harmonie les deux groupes ethnoculturels Adja et Ehoé de la Préfecture. En outre, des enquêtes préliminaires ont montré que c'est ce quartier qui comprend les paysans les plus âgés encore actifs. Enfin, à l'intérieur de ce même quartier, les ménages ont été choisis au hasard. Les chefs d'exploitation et leur(s) femme(s) ainsi que des responsables de divers services agricoles de la zone ont été les répondants.

Les données sont recueillies par des interviews semi-structurées et des observations de terrain au champ et au marché à l'aide d'un guide d'entretien. 
Les questions sont relatives aux diverses techniques utilisées pour la conservation des récoltes, à leur efficacité, etc.

Les pratiques agricoles de conservation des récoltes ont été notées selon leur modalité, leur opportunité et leur efficacité. II s'est agit d'observer et de décrire objectivement ce que le paysan fait, d'analyser la logique interne ou la rationalité de ses pratiques et d'évaluer leurs conséquences et leurs performances. Les noms des cultivars et des espèces sont recueillis en Ehoé et en Adja, les deux principales langues parlées dans la zone.

\section{TRAITEMENT DES DONNEES}

Le dépouillement des guides d'entretien a été réalisé manuellement. A l'aide de Microsoft EXCEL ${ }^{\odot}$, ces données ont servi à calculer le pourcentage d'adoption des pratiques par les paysans, c'est-à-dire le pourcentage de paysans qui adoptent une pratique et la mettent effectivement en œuvre, ce qui permet d'estimer l'importance relative de chaque pratique. Ces résultats ont été aussi soumis au logiciel STATISTICA 6.0@ pour la classification hiérarchique des exploitations.

\section{RESULTATS ET DISCUSSION}

Des récipients de diverses natures sont utilisés mais dans d'autres cas, des fosses sont aménagées pour la conservation des récoltes. Pour une production donnée, les greniers peuvent être destinés à la conservation des denrées alimentaires («Adova») ou au stockage des semences pour les campagnes agricoles à venir. Pour les légumineuses et les céréales, les systèmes de conservation diffèrent selon qu'elles sont décortiquées ou non. Les greniers de types Ava, Huidza ou Pidza, Sogba, silo et citerne, le système Adjoko, les sachets, les bidons, les fûts et les tonneaux constituent les principaux systèmes de conservation de graines et grains recensés dans la zone d'étude. Des systèmes particuliers de conservation des tubercules et racines sont aussi recensés.

\section{TYPOLOGIE STRUCTURELLE DES EXPLOITATIONS}

Le but de cette typologie est de catégoriser les exploitations en fonction de leur degré de conservation. Ce degré est apprécié par le nombre de cultivars locaux et l'existence de palmeraie dans les exploitations. La figure 2 classe les exploitations en neuf ensembles regroupés au sein de deux grandes catégories. L'analyse de cette figure permet d'obtenir l'interprétation contenue dans le tableau 1.

Le tableau 1 montre que tous les ensembles du groupe A ont sécurisé leurs exploitations par la plantation de palmeraie. En effet, dans la zone d'étude, les palmeraies constituent un moyen discret d'appropriation de la terre. En outre, plus de $80 \%$ de ce groupe disposent des deux meilleurs types de sols. Ils ont aussi le plus grand nombre de cultivars locaux (2 à 9). Par conséquent, c'est au sein de ces groupes qu'on peut manipuler le plus grand nombre de cultivars et donc, de ressources phytogénétiques.

Par contre, le manque de terre et surtout de sols fertiles est un problème dont souffrent les ensembles du groupe B. Seuls $32 \%$ de ce groupe disposent d'une palmeraie. Cela peut être lié au manque de terre. Ils disposent aussi de peu de cultivars locaux dans leurs exploitations. D'autre part, le nombre de personnes en charge reste relativement important (6). Ce groupe penserait beaucoup plus à la satisfaction immédiate des besoins familiaux qu'à la conservation des ressources phytogénétiques.

Cette analyse peut se résumer en trois points : les exploitations conservatrices représentées par les ensembles du groupe $A$ et $E_{9}$ du groupe $B$, les exploitations semi-conservatrices que forment les ensembles $E_{5}, E_{7}, E_{8}$ du groupe $B$ alors que l'ensemble $E_{6}$ peut être considéré comme des exploitations non conservatrices. $\mathrm{Ce}$ dernier ensemble regroupe des gens relativement jeunes (âge maximal : 50 ans).

La conservation des ressources phytogénétiques n'est donc pas l'apanage des gens d'un certain âge précis de la population. Cela 
peut donner moins d'inquiétude quant à l'avenir des spéculations. En effet, si cela était le cas, l'on pourrait penser qu'avec la disparition du groupe concerné, beaucoup de spéculations disparaîtraient. Toutefois, le fait que ce soit la disponibilité foncière qui soit le facteur essentiel de la conservation alors qu'une proportion importante des exploitations ne dispose pas de terres fertiles suscite beaucoup d'interrogations. Le tableau 1 montre que seuls $34,21 \%$ des exploitations possèdent encore les sols
Avédzi et/ou Ekpan. La grande majorité ne dispose que des terres pauvres. L'important taux de croissance de la population peut aussi faire progresser rapidement la proportion des exploitations non conservatrices qui est actuellement d'environ $44 \%$. Les populations déve-loppent toujours des stratégies pour conserver les récoltes, quelle que soit leur quantité, soit pour la consommation, soit pour servir de semences les saisons à venir.

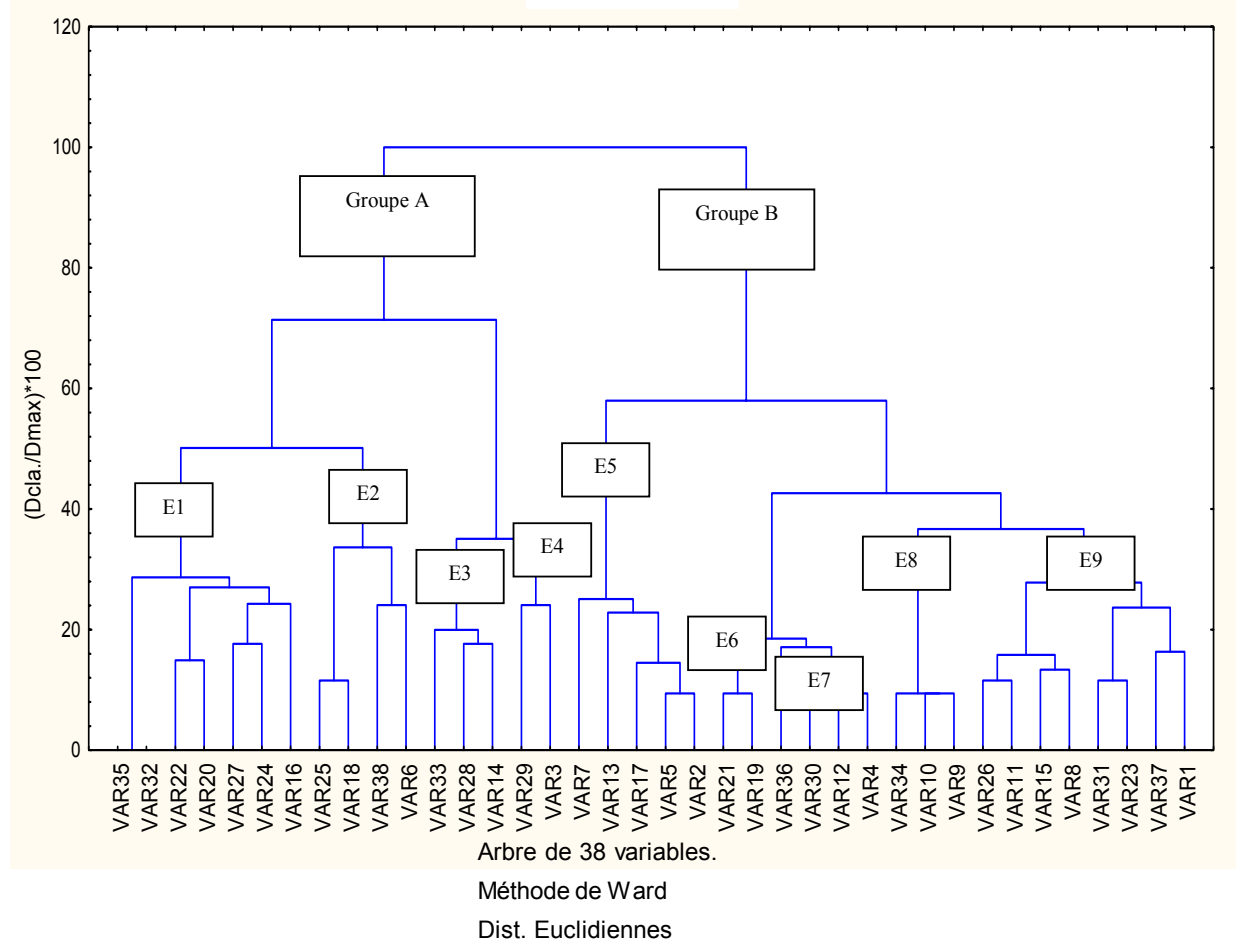

Figure 2 : Classification hiérarchique des exploitations.

Hierarchical classification of livelihoods.

Tableau 1 : Interprétation de la typologie des exploitations.

Interpretation of the structure of livelihoods.

\begin{tabular}{|c|c|c|c|c|c|c|c|}
\hline Groupe & Ensemble & $\%$ d'exploitation & $\begin{array}{l}\text { Age du chef } \\
\text { d'exploitation }\end{array}$ & Type de sol & Spéculations & $\begin{array}{l}\text { Existence de } \\
\text { palmeraie }\end{array}$ & $\begin{array}{l}\text { Personne en } \\
\text { charge }\end{array}$ \\
\hline \multirow{4}{*}{ A $(42,10 \%)$} & $E_{1}$ & 18,42 & 40 à 60 ans & Avédzi et Ekpan & $3 \mathrm{ci},+$ de $3 \mathrm{cn}, \mathrm{ml}$ & oui & 7 \\
\hline & $\mathrm{E}_{2}$ & 10,53 & 50 à 75 ans & Avédzi et Ekpan & $\mathrm{ml}, \mathrm{pp}$ & oui & 4 \\
\hline & $\mathrm{E}_{3}$ & 7,89 & 20 à 50 ans & Zoxo et Kpodzi & $\mathrm{ml}, 2 \mathrm{~cm}$ & oui & 5 \\
\hline & $\mathrm{E}_{4}$ & 5,26 & 64 à 67 ans & Avédzi et Ekpan & $\begin{array}{c}\mathrm{ml}, 2 \mathrm{~cm},+\mathrm{de} \\
3 \mathrm{cn}, \mathrm{ar}, \mathrm{pp}\end{array}$ & oui & 1 \\
\hline \multirow{5}{*}{ B $(57,90 \%)$} & $\mathrm{E}_{5}$ & 13,16 & 20 à 35 ans & Kpodzi ou Zoxo & $\mathrm{ml}, \mathrm{tl}$ & non & 5 \\
\hline & $\mathrm{E}_{6}$ & 5,26 & 20 à 50 ans & $\begin{array}{l}\text { Avédzi ou Ekpan } \\
\text { ou Zoxo ou Kpodzi }\end{array}$ & autres* & non & 4 \\
\hline & $\mathrm{E}_{7}$ & 10,53 & 51 à 75 ans & $\begin{array}{l}\text { Avédzi ou Ekpan } \\
\text { ou Zoxo ou Kpodzi }\end{array}$ & $2 \mathrm{ci}$ & oui & 7 \\
\hline & $\mathrm{E}_{8}$ & 7,89 & 51 à 75 ans & $\begin{array}{l}\text { Avédzi ou Ekpan } \\
\text { ou Zoxo ou Kpodzi }\end{array}$ & $\mathrm{ml}, 2 \mathrm{ci}$ & oui & 9 \\
\hline & $\mathrm{E}_{9}$ & 21,05 & 40 à 70 ans & $\begin{array}{l}\text { Avédzi ou Ekpan } \\
\text { ou Zoxo ou Kpodzi }\end{array}$ & $\mathrm{ml},+$ de $3 \mathrm{cn}, 1 \mathrm{ci}$ & non & 3 \\
\hline
\end{tabular}

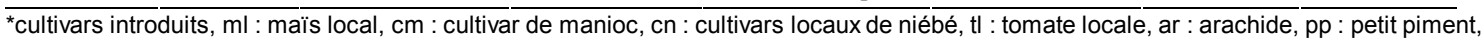
ci : cultivars d'igname. 


\section{PRATIQUES DE CONSERVATION DES RECOLTES ET SEMENCES}

\section{Conservation du maïs}

Plusieurs systèmes interviennent dans la conservation du maïs. II s'agit essentiellement des greniers de types Huidza ou Pidza (53\%), Ava (29\%), Sogba (13\%) et citerne (8\%), du système Adjoko ( $8 \%$ ) mais aussi des silos $(5 \%)$. Les résultats de nos études sur le taux d'adoption des diverses pratiques montrent que les greniers de type Pidza et Ava sont les plus fréquemment utilisés pour conserver le maïs non déspathé (Figure 3).

Le grenier de type Ava (Figures 4a et 4b) est une structure circulaire dont le diamètre varie d'un à quatre mètres selon l'importance de la récolte. Des piliers solides sont disposés sur deux cercles; leur écartement varie suivant leur grosseur, qui elle même, dépend de l'abondance de la récolte. Plus ils sont gros, moins ils sont rapprochés les uns des autres. Sur cette structure, on construit une plateforme conique vers le bas sur lequel la récolte de maïs est disposée. On recouvre l'ensemble d'une toiture de paillassons d'Imperata cylindrica (L.) Raeuschel. Cette couverture peut également être en plastique ou en tôle ondulée. Les piliers sont souvent tirés d'espèces à bois dur et résistant à la pourriture et aux termites comme Millettia thonningii (Schum. \& Thonn.) Bak., Borassus aethipium Mart., Azadirachta indica A. Juss. et Fagara zanthoxyloides (Lam.) Zepernick \& Timler. Leur hauteur est variable suivant qu'on soit au champ (Figure 4a) ou à domicile (Figure 4b). Le système installé, plus particulièrement les piliers, peut servir pendant trois à quatre saisons. Le grenier du type Ava, un des plus anciens systèmes endogènes de conservation, est beaucoup plus utilisé dans les milieux où la divagation des ovins et des caprins est contrôlée. II permet une meilleure conservation des ressources naturelles mais aussi des savoirs qui leur sont liés (IPGRI, 1999; Engels et al., 2001)

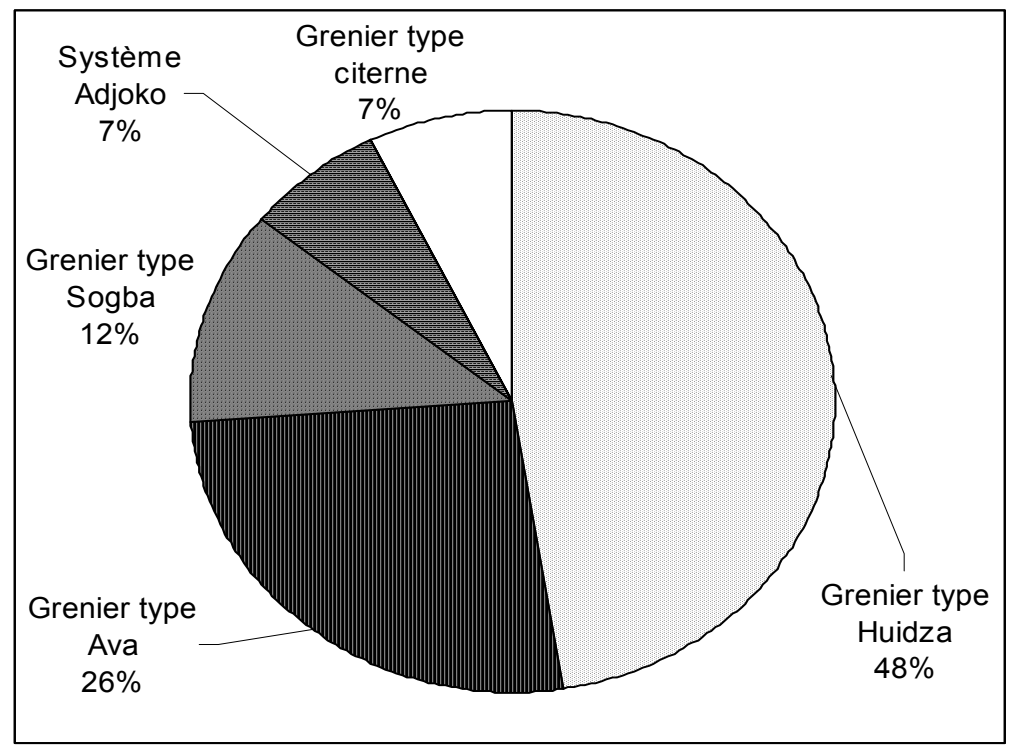

Figure 3 : Importance relative des pratiques de conservation de maïs.

Relative importance of the practices of maize harvesting conservation.

Le grenier du type Huidza (Figure 4c) est une sorte de grand panier sans fond dont le diamètre peut atteindre 1,5 $\mathrm{m}$ et la hauteur, $2 \mathrm{~m}$. II est confectionné avec des tiges de Mallotus oppositifolus (Geisel) Müll. Arg., A. indica, M. thonningii ou Bambusa vulgaris Schrader ex Wendel. Rempli de maïs, il est couvert d'un paillasson d' l. cylindrica ou d'une toiture en tuile ou encore de plastique. Le grenier de type Huidza, beaucoup plus moderne, protège les récoltes contre les voleurs et les animaux (ovins, caprins, etc.). Il contribue plus à la dégradation de l'environnement que ne le font les autres types de greniers car les prélèvements de matériel végétal pour sa confection sont plus importants. Malgré tout, il reste le type de grenier le plus 
utilisé (Figure 3). Ces faits corroborent les observations de Jouve (1997) selon lesquelles les choix des agriculteurs ne sont pas toujours les meilleurs possibles pour la communauté ni pour la protection de l'environnement.

Le grenier du type citerne, de dimensions variables (3 à $4 \mathrm{~m}$ de diamètre et $1 \mathrm{~m}$ de profondeur), est surélevée d'une margelle de 0,5 à $1 \mathrm{~m}$ de hauteur (Figure $4 \mathrm{~d}$ ). II est peu utilisé (Figure 3) et est semblable au grenier du type Ava. La faible hauteur de la margelle peut exposer les récoltes aux attaques des animaux. Pour cette raison, il est toujours installé au champ. Cette faiblesse couplée à son coût élevé (par l'achat du ciment) explique sa faible utilisation.

Le grenier du type Sogba est un apatam fait de quatre piliers sous lequel on installe une cuisine ; les récoltes sont entreposées sur le toit puis couvertes (Figure 4e). II prend peu de place et facilite la conservation par la chaleur et les fumées produites par le feu de la cuisine. II est fait pour les petites récoltes de maïs, pour le niébé ou pour le sorgho et demande peu de moyens. Cet exemple est très proche du système Rumbu des peuples Aten de Ganawuri au Nigeria pour conserver le fonio et du système Kiro des peuples Moru du Sud Soudan dans la conservation du sorgho (IPGRI, 1994).

La conservation par le système Adjoko est surtout une conservation de semences de maïs pour les prochaines cultures (Figure 4f). Les semences sont accrochées au toit de la cuisine, au-dessus du foyer. Elles bénéficient ainsi de la fumée et de la chaleur et le séchage peut ainsi se poursuivre. Les semences déjà égrenées sont emballées par des tissus de coton, puis accrochées au toit. Dans la Préfecture de Yoto, voisine de celle du Moyen-Mono, Datchidi (2002) a montré que la position du maïs par rapport à la source du feu varie en fonction de son état physiologique à la récolte. Plus, il est proche de la maturité, plus il est éloigné de la source du feu. Le système Adjoko, de moins en moins pratiqué, n'existe encore que pour la conservation de semences d'espèces rares ou lorsque l'on veut utiliser la récolte de la première saison comme semence pour la seconde saison. C'est ce qui explique son faible taux d'adoption (Figure 3).

Les systèmes de conservation décrits sont utilisés pour conserver du maïs non égrené ou mieux, avec ses spathes. Par contre, le maïs égrené se conserve dans des tonneaux ou «silos» (Figure 4g). Ils sont très peu adoptés car les paysans préfèrent conserver rapidement le maïs avec ses spathes. L'adoption du silo et de la citerne, quoique financièrement coûteuse, peut constituer des solutions écologiques car n'ayant pas d'impact négatif majeur sur l'environnement. Par ailleurs, le coût élevé de ces types de greniers est largement compensé par leur durabilité temporelle.

Enfin, la plupart des paysans préfèrent séparer le stock de consommation de celui destiné aux semences.

La conservation du maïs est renforcée par l'utilisation des pesticides biologiques et chimiques. Ainsi, au moment de la mise en place du grenier, on asperge par étages successifs, divers produits variant en fonction de l'exploitant et suivant les moyens, de l'extrait de neem, aux pesticides de synthèse, en passant par le pétrole mélangé au sable, à l'eau salée, au sel de cuisine ou à la cendre. II faut reconnaître que l'utilisation de l'extrait de neem, méthode biologique, est encore à son début dans la zone. Elle mérite d'être encouragée du fait de son efficacité prouvée contre les déprédateurs (Mazhar, 1999; IPGRI, 2001).

Les études ont prouvé que la majorité des paysans (soit $50 \%$ ) préfèrent utiliser des produits phytosanitaires initialement destinés aux traitements du cotonnier malgré l'illégalité de cette pratique et ses effets sur la santé humaine et l'environnement, des faits bien reconnus par les paysans (Tableau 2). Ces produits phytosanitaires sont souvent mélangés au sable fin, à la cendre ou aux sciures de bois avant leur application aux récoltes. Les paysans pensent que ce faisant, ils évitent le contact direct entre le produit et les récoltes, minimisant ainsi les risques d'intoxication. Cependant, ce fait est loin de concilier les objectifs de préservation de la santé et la conservation. En effet, dans un travail de conservation, le «conservationiste» doit clairement comprendre et démontrer les liens entre la santé et la biodiversité afin de promouvoir la conservation comme un moyen de protection et d'amélioration de la qualité de la vie humaine (WWF, 2001).

Certains paysans font le grenier du type Huidza sans utilisation de produits mais en jouant sur le temps de la récolte. Ils estiment qu'en récoltant le maïs un peu plus tôt et en conservant la récolte dans le grenier du type Huidza, on emprisonne le peu d'eau que la récolte contient 
encore, ce qui produirait une chaleur qui protège la récolte. La réussite de ce système éviterait les ennuis de santé que pourraient provoquer la consommation de denrées conservées avec les produits phytosanitaires. Ce serait la promotion

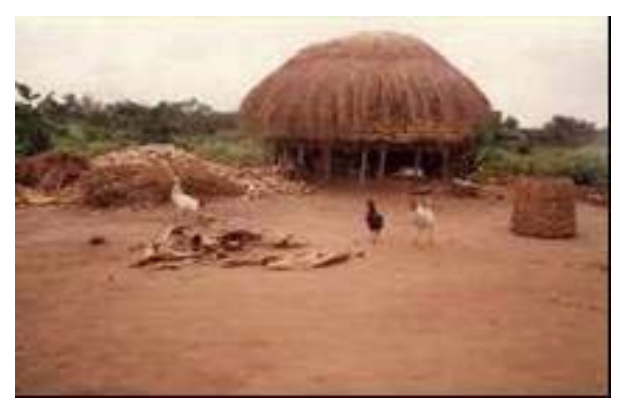

a - Grenier type "Ava" dans les champs.

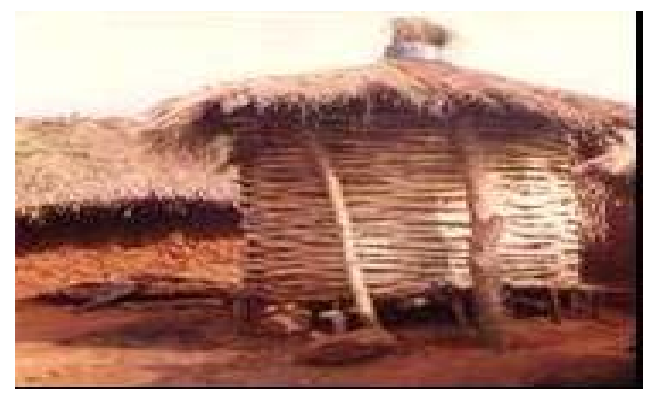

c - Grenier type Huidza fait avec des lattes de bambou (Bambusa vulgaris).

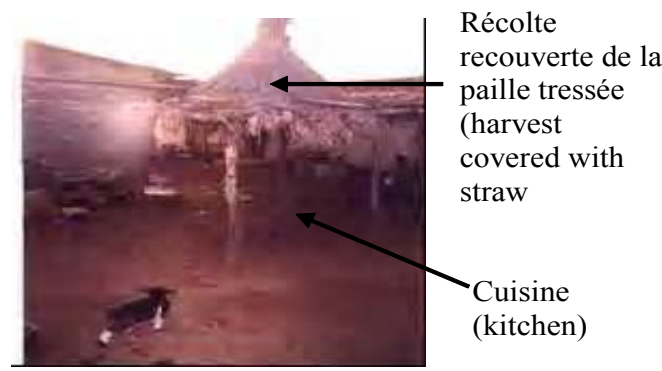

e - Grenier type Sogba. d'une agriculture saine pour l'environnement et la santé (IPGRI, 1999 ; Engels et al., 2001). Le tableau 2 résume les taux d'adoption de ces différents produits par les paysans pour la conservation du maïs.

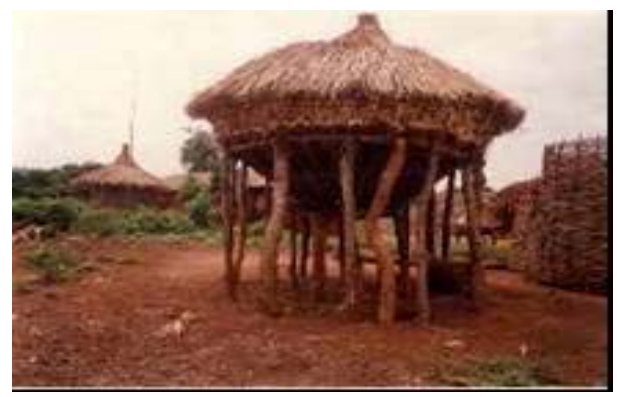

b - Grenier type "Ava" à la maison

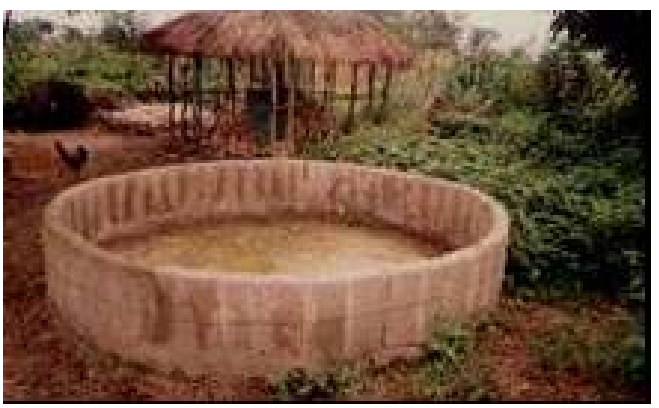

d - Grenier type citerne.

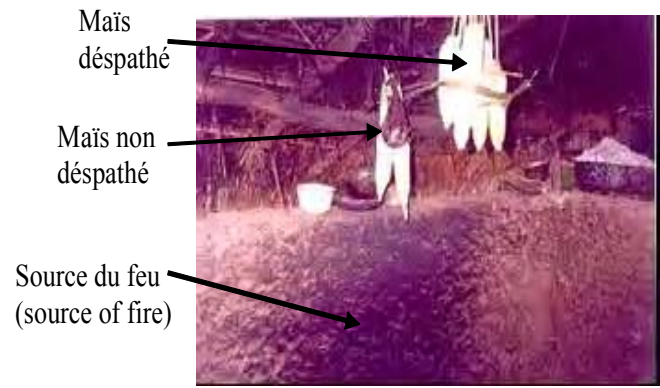

f - Un dispositif du système Adjoko.

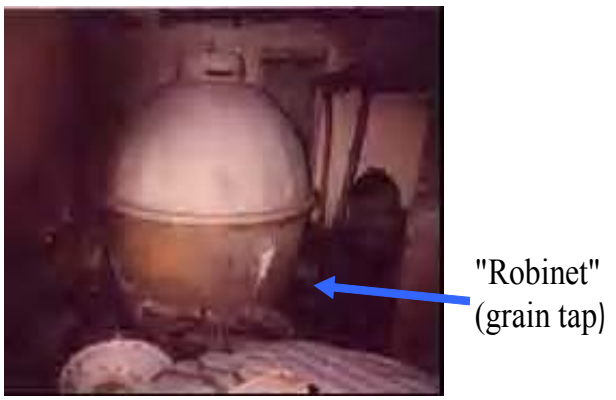

g - Grenier type silo

Figure 4 : Types de greniers et dispositifs du système Adjoko.

Granaries and Adjoko system. 
Tableau 2 : Produits utilisés pour renforcer la conservation.

Products using to strengthen the conservation.

\begin{tabular}{lc}
\hline Produits de conservation & $\begin{array}{c}\text { Taux d'adoption } \\
\text { par les paysans (\%) }\end{array}$ \\
\hline Cendre (ash) & 26,32 \\
Extrait de neem (juice of Azadirachta indica) & 21,05 \\
Sable fin (sand) & 10,05 \\
Grain de sel de cuisine (grain of kitchen salt) & 10,05 \\
Pétrole (petrol) & 5,26 \\
Sciures de bois (sawdust) & 2,63 \\
Produits phytosanitaires (synthetic products) & 50,00 \\
\hline
\end{tabular}

\section{Conservation du niébé}

Le niébé se conserve essentiellement égrené, souvent en mélange avec les résidus des gousses, dans divers contenants tels que sachets, bidons, fûts, tonneaux, silos, etc. (Figure 5). Cette stratégie est adoptée par $54 \%$ des exploitations. Ce système de conservation est renforcé chez $24 \%$ des exploitations par l'utilisation du péricarpe séché d'orange et/ou du piment (Capsicum sp.) bien séché. Ces additifs jouent le rôle de véritables biopesticides contre les larves de charançons (Mazhar, 1999 ; IPGRI, 2001). Selon les paysans, le péricarpe séché d'orange et le piment permettent d'obtenir de très bons résultats. L'efficacité de ce système est liée à l'émission de substances volatiles répulsives qui empêchent l'attaque par les ravageurs. De plus, les résidus de gousses du niébé favoriseraient l'absorption de l'humidité résiduelle dans le milieu le rendant plus sec. Cela pourrait empêcher la putréfaction, source d'attaques par les bactéries et autres agents destructeurs.

Quand le niébé n'est pas décortiqué, il est conservé dans le grenier Sogba ou quelquefois par Adjoko ou encore dans le grenier du type Huidza (Figure 5). Ces résultats montrent que le niébé se conserve très mal avec les gousses. Cependant, la fumée et la chaleur de la cuisine peuvent contribuer à cette conservation. C'est ce qui explique la proportion relativement importante des utilisateurs du grenier du type Sogba.

\section{Conservation des tiges du manioc}

Le manioc se multiplie par boutures ; aussi ses tiges se conservent-elles par la mise en terre directe des boutures au moment de la récolte ou par la protection des bottes de tiges par des branchages (Figure 6). La mise en terre directe des boutures est pratiquée si la récolte coïncide avec la saison des pluies et/ou la bonne saison de plantation. Par contre, la mise des bottes de tiges contre tuteurs (palmiers à huile le plus souvent) ou couchées à même le sol dans un milieu humide en brousse sont des stratégies adoptées Iorsque l'on veut garder les «semences» en attendant la bonne saison de plantation. En mettant les bottes de tiges contre tuteur, le paysan les met du côté ouest de celuici ; car l'ensoleillement de la matinée est estimée trop intense. De plus, toute la partie distale en contact avec les tubercules est laissée intacte afin d'éviter la perte des réserves accumulées dans cette partie de la tige. Enfin, on amasse de la terre à la base de la botte pour favoriser la conservation de l'humidité sous les tiges. Malgré ces précautions, il se produit souvent un dessèchement des tiges dans leur partie supérieure suite à la migration de l'eau vers le bas. Cette stratégie reste néanmoins la plus adoptée par les exploitations (Figure 6).

Quand les bottes de tiges sont couchées à même le sol dans un endroit ombragé, les tiges sont mieux conservées et poussent même des racines. Cette méthode est néanmoins peu appréciée par les paysans car les tiges sont exposées aux déprédations et, une fois mises en terre, la levée des boutures n'est pas facile.

Les «semences» peuvent être conservées à l'ombre à la maison; cette méthode s'applique aux espèces rares de manioc dont les tubercules sont très appréciés. Par ailleurs, les «semences» peuvent être conservées au champ à travers des pieds sous lesquels, on enlève de temps en temps, des tubercules, en attendant la bonne saison. Cette méthode est pratiquée 
par $8,51 \%$ des exploitations pour les espèces en disparition. Ce faible taux peut s'expliquer par le fait que les "semences» de manioc ne constituent pas souvent un problème majeur pour le paysan.

\section{Conservation des semences d'igname}

Les ignames susceptibles de servir à la préparation de l'igname pilée ou "foufou», encore appelées ignames «blanches», sont récoltées en deux temps. Elles sont sevrées en août et le reste est déterré entre novembre et décembre. Le sevrage est, en réalité, une stratégie de multiplication des têtes d'ignames. En effet, les ignames qualifiées de «blanches», ne se multiplient que par la partie distale de leurs tubercules contrairement aux ignames dites de cossettes. Ainsi, une igname sevrée peut régénérer une dizaine de "têtes» qui serviront de semences. Lorsque le tubercule est petit, il est laissé dans la butte et il régénère des semences.
La conservation dans une clôture à la maison est la stratégie la plus pratiquée (Figure 7). II s'agit d'un enclos de palmes et d'arbustes qui serviront d'ombrage pour une meilleure conservation de la récolte. Les tubercules sont disposés serrés les uns contre les autres, le bout distal vers le sol.

La mise en fosse des tubercules au champ est une stratégie utilisée seulement pour conserver des tubercules qui serviront de semences. Ainsi, à la récolte, les tubercules qui serviront de semences à la prochaine campagne, sont laissées soit dans leurs buttes respectives, soit dans une fosse commune creusée à cet effet. Cette stratégie est adoptée dans le cas de mauvaises récoltes mais aussi, dans le cas où les champs sont très loin des habitations. Elle vise à réduire les efforts pour le transport et à dérober les semences des besoins alimentaires. Dans d'autres cas, les tubercules sont conservés dans un milieu frais et recouvert de détritus ou dans un grenier du type Ava. Ces deux méthodes, peu conservatrices, sont de loin les moins adoptées.

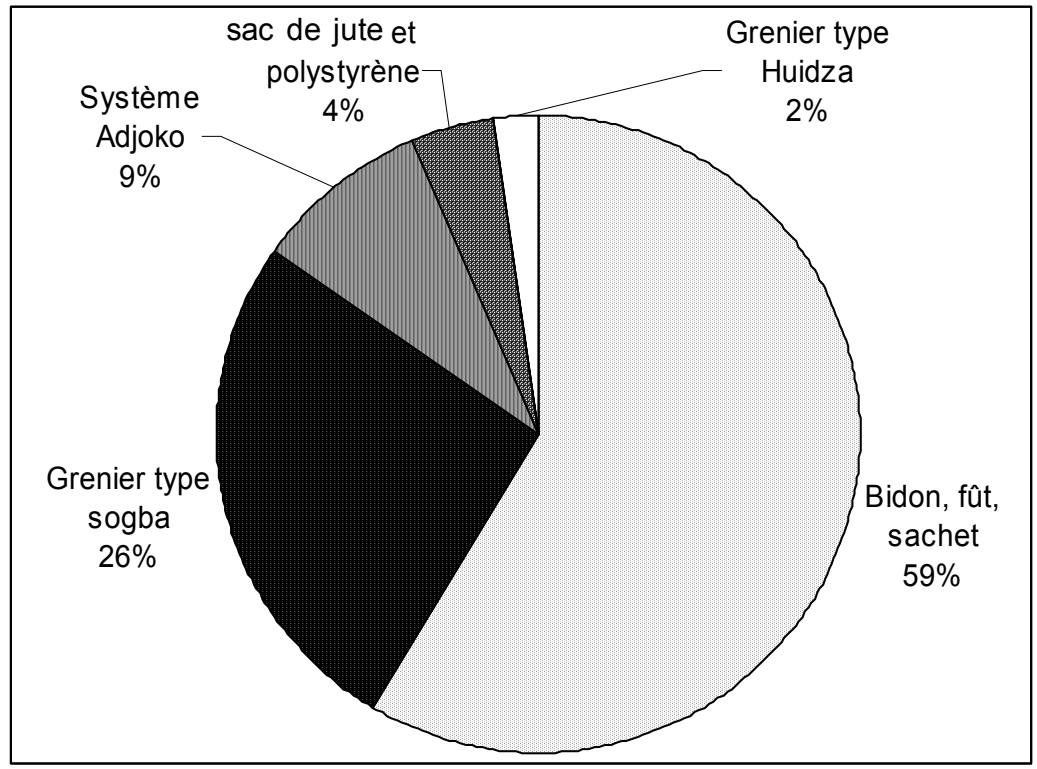

Figure 5 : Importance relative des pratiques de conservation du niébé.

Relative importance of the practices of cowpea harvesting conservation. 


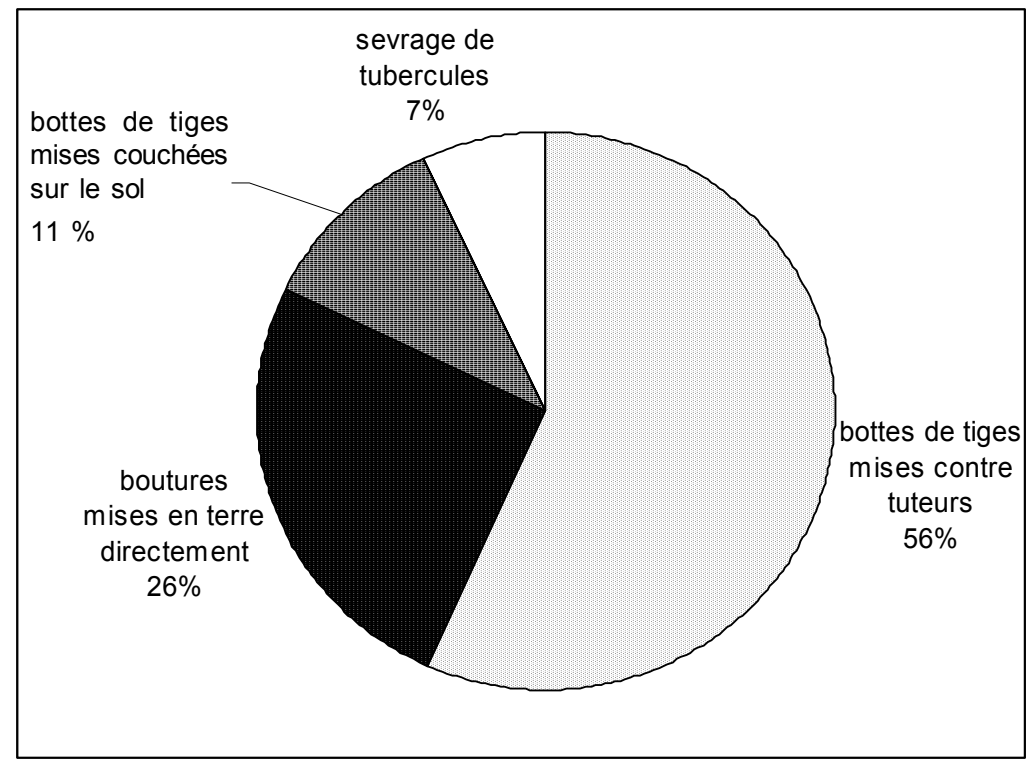

Figure 6 : Importance relative des pratiques de conservation du manioc.

Relative importance of the practices of top boots of cassava conservation.

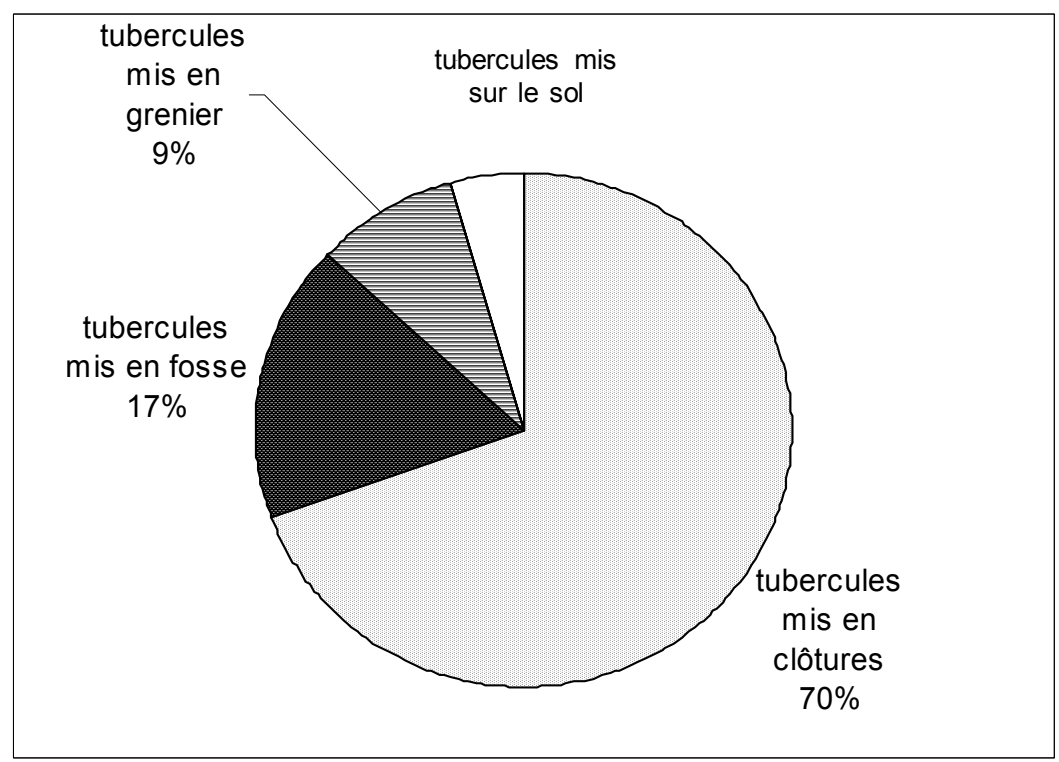

Figure 7 : Importance relative des pratiques de conservation des semences d'igname.

Relative importance of the practices of yam seeds conservation.

\section{CONCLUSION}

L'utilisation des greniers de types «Huidza» et "Ava» pour le maïs, la mise en bidons et fûts pour le niébé constituent les pratiques de conservation de semences couramment observées dans la Préfecture du Moyen-Mono au Togo. Des produits comme la cendre, le jus d'Azadirachta indica, le sable fin, les grains de sel de cuisine, les sciures de bois et le péricarpe sec de Citrus sp. et/ou du Capsicum sp. sont 
utilisés dans le processus de renforcement de cette conservation. Pour le manioc, les bottes de tiges sont mises contre tuteur du côté ouest de celui-ci alors que les semenceaux d'ignames sont conservés dans une clôture en milieu ombragé à domicile.

Les pratiques de conservation des récoltes recensées restent informelles et très variées. Friis-Hansen and Sthapit (1999) ont montré qu'elles constituent les meilleures sources d'approvisionnement en semences dans la plupart des pays en développement en particulier pour les cultures vivrières. Elles sont essentiellement liées aux types de culture et à la quantité de la récolte. L'adoption des pratiques de conservation par les paysans est conditionnée par certains facteurs : les possibilités financières, la saison de récolte et la situation du champ par rapport au village sont les plus importantes. Des études ultérieures évaluant le coût/bénéfice de ces pratiques de conservation seraient nécessaires pour mieux appréhender leur efficacité.

\section{REFERENCES}

Akpavi S. 2003. Savoirs locaux et gestion des ressources phytogénétiques dans les systèmes de culture dans le Moyen-Mono au Togo. Niamey, CRESA/FA/UAM, 85 p.

Anonyme. 2002. Monographie de la préfecture du Moyen-Mono Tohoun, Préfecture du MoyenMono, $3 \mathrm{p}$.

Brunel J. F., Hiekpo P. et H. Scholz. 1984. La flore analytique du Togo: Phanérogames. Eschborn, GTZ, 684 p.

Datchidi K. M. 2002. Stratégies paysannes de gestion des ressources phytogénétiques cultivées dans la préfecture de Yoto au Togo. Niamey, CRESA/FA/UAM, 71 p.

DSID. 1998. Caractéristiques structurales de l'agriculture togolaise. Rapport principal. Direction de la statistique. Lomé, Direction des Statistiques Agricoles, de l'Informatique et de la documentation, $113 \mathrm{p}$.

Engels J. M. M., Withers L., Raymond R. and H. Fassil. 2001. The importance of PGR and Strong national programme. In J. M. M. Engels , R. Vodouhè, J. Thompson, A. Zannou, E. Hehne and M. Grum (Eds.). Proceedings of an international workshop :
Towards Sustainable National Plant Genetic Resources Programs, Policy, Planning, and Coordination Issues. Zschortau, DSE, 11 - 21

Engels J. M. M. 2001. Complementary strategy for improved conservation and use of PGR. In J. M. M. Engels, R. Vodouhè, J. Thompson, A. Zannou, E. Hehne and M. Grum (Eds.). Proceedings of an international workshop: Towards Sustainable National Plant Genetic Resources Programs, Policy, Planning, and Coordination Issues. Zschortau, DSE, 69 - 77.

Friis-Hansen E. and B. Sthapit. 1999. Overview, local PGR management and participatory crop improvement. In E. Friis-Hansen and B. Sthapit (Eds.). Participatory approaches to the conservation and use of plant genetic resources, Rome, IPGRI, 84 - 89.

Gnamkoulamba A. 2001. Problématique de la domestication d'une espèces d'igname sauvage : Bayere (Dioscorea praehensilis) à Agbokopé (Préfecture de Wawa au Togo). Niamey, CRESA/FA/UAM, $72 \mathrm{p}$.

Groupe Crucible II. 2001. Le débat des semences. Vol. 1. pp 9 - 25.

IPGRI. 1994. Focus on West Asia and North Africa ; Geneflow Debate biodiversity prospecting : repatriating genetic resources to war-torn countries. Rome, IPGRI, 20 p.

IPGRI. 1999. Diversity for development. The new strategy of the International Plant Genetic Resources Intitute, Sthrengtening national systems. Rome, IPGRI, 15 p.

IPGRI. 2001. Regional Report Sub-Saharan Africa 1999 - 2000. Rome, IPGRI, 27 p.

Jouve P. 1997. Des techniques aux pratiques; Conséquence méthodologique pour l'étude des systèmes de production agricoles et le développement rural. In C. DortheMonachon et G. Clivaz (Eds.). Méthode pour mesurer les pratiques agraires en milieu tropical et leur transformation. Travaux et recherche de I'IGUL. Lausanne, IGUL, $101-114$

Kakabou B. 1991. Contribution à l'étude des méthodes traditionnelles de conservation des ignames. Lomé, ESA, Univ. Lomé, 53 p.

Knoth J. 1993. Stockage traditionnel de l'igname et de manioc et son amelioration. Eschborn, GTZ, $59 \mathrm{p}$.

Mazhar F. 1999. Seeds conservation and management : participatory approaches of 
Najakrichi seed network in Bangladesh. In E. Friis-Hansen and B. Sthapit (Eds.). Participatory approaches to the conservation and use of PGR, establishing seeds banks improving seed systems. Rome, IPGRI, $149-153$.

Weiskopf B. 2001. GTZ sectorial projet : managing agrobiodiversity in rural areas. In Proceedings of an international workshop :
Towards Sustainable National Plant Genetic Resources Programs, Policy, Planning, and Coordination Issues. 10 - 18 May 2000, Zschotau, Germany : pp $112-115$.

WWF. 2001. Announce of prevention : making the link between health and conservation. Doing conservation better. Washington DC, $58 \mathrm{p}$. 